\title{
Preferencia y popularidad: patrones diferenciales por género en los correlatos del alto estatus
}

\author{
Irene Jiménez-Lagares $^{1 *}$, Victoria Muñoz Tinoco ${ }^{1}$, Tamara García $^{1}$, Carolina Florindo ${ }^{2}$ \\ ${ }^{1}$ Universidad de Sevilla ${ }^{2}$ Universidad Jaume I
}

(Recibido 14 Marzo Marzo 2011, Aceptado 13 Abril 2011)

RESUMEN: Este trabajo investiga las diferencias de género en dos dimensiones del estatus social en el grupo, la preferencia social y la popularidad percibida, en una muestra de 274 adolescentes de 11 aulas de $3^{\circ}$ y $4^{\circ}$ de la ESO (119 chicos, 155 chicas) de dos centros de la provincia de Sevilla. En primer lugar, se ponen en relación las dos dimensiones de estatus con diversos atributos personales; en segundo lugar, se analizan las diferencias de género asociadas a esta estructura relacional. Los resultados muestran patrones de correlación que diferencian la preferencia social y la popularidad, así como patrones correlaciónales diferentes por género para cada una de las dimensiones analizadas.

Palabras clave: preferencia social, popularidad percibida, reputación entre iguales, agresión, diferencias de género

Preference and popularity: differential patterns by gender in the correlates of high status

ABTRACT: This study explores gender differences in two dimensions of social status in the group, social preference and perceived popularity in a sample of 274 adolescents from 11 classes of 3 and 4 of the ESO (119 boys, 155 girls) from two schools in the province of Seville. First, put on the two dimensions of status with various personal attributes, and secondly, we analyze gender differences associated with the relational structure. The results show correlation patterns that differentiate the social preference and popularity, as well as different correlational patterns by gender for each of the dimensions analyzed.

Keywords: social preference, perceived popularity, peer reputation, gender differences

La posición social en el grupo es un tema al que niños y niñas, y especialmente adolescentes parecen dedicar tiempo y atención. Pero, ¿qué significa tener una buena posición en el grupo? ¿Ser querido por los demás, tener muchos amigos, tener poder sobre las relaciones? Para Lease, Kennedy y Axelroad (2002), la pregunta ¿Qué significa ser popular? solo tiene una respuesta posible: depende de a quién se le pregunte y de cómo se formule la pregunta. Así, investigadores de distintas tradiciones han respondido de modo distinto, y han preguntado de modos diversos.

Desde la sociometría tradicionalmente se utilizaba el término popular para referirse a aquellos chicos y chicas que gustaban a sus compañeros, técnicamente, 
aquéllos que, ante la pregunta ¿Con quién te gusta estar? recibían gran cantidad de nominaciones positivas y pocas negativas. Parecía bien establecido que estos populares sociométricos, por así llamarlos, eran más simpáticos, cooperativos, prosociales y habilidosos socialmente que sus compañeros (Newcomb, Bukowski y Pattee, 1993). Sin embargo, la investigación de corte más sociológico sugería que los adolescentes populares eran atractivos, socialmente dominantes y habilidosos socialmente, pero no necesariamente gustaban a sus compañeros, ni eran tan cooperativos y prosociales como podía deducirse de la investigación sociométrica. De hecho, si se sustituye la pregunta anterior por ¿Quién es la persona más popular de tu grupo?, el perfil de los elegidos cambiaba sustancialmente. Aparecen así los populares duros o modélicos de Rodkin, Farmer, Pearl y Van Acker (2000), o los populares prosociales o antisociales de Bruyn y Cillessen (2006a) o los líderes convencionales y líderes no convencionales de Miller-Johnson, et al. (2003).

Fue consolidándose entonces la idea de que era necesario diferenciar a los chicos y chicas preferidos sociométricamente de aquéllos que son percibidos como populares (Andreou, 2006, Estell,Farmer, Pearl, Van Acker, Rodkin, 2003, García-Bacete, 2008), es decir, que la preferencia social y la popularidad no son constructos redundantes (Cillessen y Rose, 2005). No obstante, preferencia y popularidad están positivamente relacionadas, y existe un solapamiento entre chicos y chicas preferidos y populares.

Para describir los perfiles conductuales y personales de preferidos y populares lo más frecuente ha sido acudir a la evaluación de los iguales. La reputación entre iguales hace referencia al conjunto de juicios que una comunidad realiza acerca de las cualidades personales de uno de sus miembros. Desde esta perspectiva, la reputación es un fenómeno colectivo producto de procesos sociales (Emler, 1990). Al tratarse de una medida de valoración por parte de los iguales, la reputación conductual presenta ciertas ventajas frente a otros modos de estudiar la conducta social. Para Rubin, Bukowski y Parker $(1998,2006)$ estas ventajas residirían, en primer lugar, en que las características que los iguales determinan como importantes serán las que el propio grupo utilizará para la inclusión o la exclusión. Además, la evaluación de iguales incluye necesariamente la perspectiva de muchos observadores, con lo que se minimizan los posibles errores relacionados con características relacionales idisiosincrásicas (peleas, rencillas escolares, etc.). Por último, la evaluación de los iguales suele estar basada en un amplio rango de experiencias y situaciones distintas, puesto que los compañeros suelen tener un acceso privilegiado a muchas conductas que no son fácilmente observables para otros informantes, como la familia o el profesorado, lo que ofrecería una evaluación diversa y matizada. El grupo de iguales se convierte, por tanto, en una inestimable fuente de datos acerca de las relaciones sociales que en su seno se establecen (Parker y Asher, 1987) y provee de un índice de competencia social muy estable a lo largo de los años escolares (Coie, Dodge y Kupersmidt, 1990).

En las investigaciones más recientes que incorporan esta dimensión existe 
una tendencia creciente a no utilizar exclusivamente indicadores globales, típicamente sociabilidad, aislamiento y agresión, incluyendo elementos específicos como los distintos tipos de agresión (relacional, abierta...), de aislamiento (voluntario, ligado a la ansiedad de las relaciones sociales...) o dimensiones específicas de la sociabilidad (conducta prosocial, liderazgo...). Es por ello que en este trabajo se ha optado por utilizar este último tipo de indicadores, denominados por Newcomb et al. (1993) y Gest, Sesma, Masten y Tellegen (2006) de banda estrecha, no utilizando los indicadores globales, o de banda ancha.

Pero la reputación es algo más que conducta social evaluada por observadores privilegiados. Se trata un fenómeno grupal producto de complejos procesos sociales que se comporta también como un factor socializador en sí mismo, puesto que la percepción que el grupo tiene de cada uno de sus miembros actúa como fuerza socializadora a través de las etiquetas más o menos explícitas con las que cada niño y niña es categorizado. Es decir, el concepto de reputación incluye un sesgo potencial que deriva de que, una vez establecida la reputación conductual, puede ser difícil de modificar, $\mathrm{y}$, de algún modo, se hace menos dependiente de las conductas reales que los individuos ponen en marcha (Hymel, Wagner y Butler, 1990). Así, la reputación puede estar impregnada de elementos muy salientes, aunque infrecuentes, o de conductas que fueron frecuentes, pero que en el momento actual no lo son tanto. Además, la evaluación de la reputación de otros está influenciada por diversas características, como el estatus, la edad o la simpatía hacia el otro, y, entre estas características, se encuentra la propia reputación.

Aunque por las características de este trabajo se utiliza la evaluación de iguales como una medida de evaluación de la conducta social con las ventajas ya enumeradas, no debe perderse de vista la dimensión reputacional de la misma, esto es, las características evaluadas por los iguales serán predictoras del estatus, pero también se verán mantenidas, consolidadas, y con no poca frecuencia enfatizadas, merced a dicho estatus.

Pero, ¿cuáles son estas características relevantes?, ¿cómo son vistos por los iguales los chicos y chicas preferidos y populares? Aunque se hace patente la necesidad de trabajos que sistematicen los resultados obtenidos, pueden irse definiendo distintos perfiles asociados a una y otra dimensión. Así, los chicos y chicas preferidos, como ya avanzábamos, son vistos como como prosociales, académicamente implicados y con habilidades sociales, mientras que los populares presentarían mayores niveles de agresión, especialmente relacional, y serían socialmente dominantes, es decir, tendrían acceso y control a los recursos grupales (de Bruyn y Cillessen; 2006 a y b; Hawley, 1999; Newcomb, Bukowski y Pattee, 1993; Lease, Kennedy y Axelroad, 2002).

Existen aún pocos trabajos centrados en las diferencias de género en las diversas dimensiones del alto estatus, pero todo indica que existirían perfiles diferentes para chicos y chicas en cada una de las dos dimensiones estudiadas. Así, los tipos concretos de agresión, el compromiso académico, las habilidades atléticas o la conducta antisocial son variables en las que suelen encontrarse diferencias 
ligadas al género (de Bruyn y Cillessen, 2006a; Lease et al., 2002).

En nuestro entorno no existen apenas trabajos de investigación centrados en estas dimensiones del estatus, por lo que éste que se presenta es un trabajo inicial, con objetivos más descriptivos que explicativos. Se persiguen diversos objetivos que generan también diversas hipótesis. Nuestra primera hipótesis es que preferencia y popularidad percibida estarán relacionadas, es decir, correlacionarán positivamente. Nuestro segundo objetivo consiste en analizar las correlaciones entre el conjunto de variables evaluadas $y$, respectivamente, preferencia social y popularidad percibida, hipotetizando que preferencia y popularidad percibida son fenómenos muy relacionados, pero con diferencias relevantes. En tercer lugar, se analiza el patrón de correlaciones por género, hipotetizando que encontraremos diferencias de género relevantes.

\section{MÉTODO}

\section{Participantes y procedimiento}

Participaron en el estudio 274 estudiantes de 11 aulas de $3^{\circ}$ y $4^{\circ}$ de la ESO (43,4\% chicos, $56,6 \%$ chicas)de dos centros de la provincia de Sevilla.

La dirección de los centros seleccionados recibió con antelación los instrumentos de evaluación, obteniéndose el permiso de los mismos. Miembros del equipo de investigación, o de la propia dirección del centro, explicaron al alumnado los objetivos de la investigación. Se les garantizó la confidencialidad y, dada la naturaleza de las cuestiones planteadas, se les señaló explícitamente que podían dejar cuestiones en blanco.

\section{Instrumentos. Cuestionario de nominaciones}

Los estudiantes respondieron a un cuestionario de nominaciones entre iguales con 27 ítems y cinco nominaciones posibles entre compañeros y compañeras de su aula. Para facilitar estas nominaciones, se facilitó a cada adolescente una lista de alumnos del aula. Estos ítems evaluaban: (a) aceptación social(“Alguien con quien te gusta mucho estar") y rechazo social ("Alguien con quien no te gusta mucho estar"); (b) popularidad ("Alguien con quien todo el mundo quiere estar, la persona más popular de la clase”) e impopularidad ("Alguien con quien nadie quiere estar, la persona menos popular del grupo"); (c) prerrogativas de estatus. Siguiendo las propuestas de Lease, Musgrove y Axelroad (2002), se incluyeron entre estas prerrogativas influencia ("Alguien con mucha influencia en el grupo, a quien los demás escuchan"), control social ("Alguien que tiene el control de las relaciones, que decide quién está y quién no en el grupo"), admiración ("Alguien a quien la gente admira y les gustaría ser como esa persona") y liderazgo ("Alguien a quien los demás consideran líder, y suelen dejar que tome las decisiones y las riendas de diversos asuntos"); (d) características personales y conductuales. Se realizó una selección de ítems basada en las propuestas de Lease, Musgrove 
y Axelroad (2002), el metaanális de Newcomb, Bukowski, y Pattee, (1993) y otros indicados por los objetivos específicos de este trabajo. Se incluyó: ayuda ("Alguien que ayuda a los demás cuando lo necesitan, que siempre echa una mano"), tristeza (“Alguien que casi siempre parece triste”), transgresión de las normas ("Alguien que no sigue las reglas") (por ejemplo, replica o desobedece a los profesores), inteligencia ("Alguien inteligente, que sabe muchas cosas"), disrupción ("Alguien que interrumpe a los otros, o se entromete cuando los demás están trabajando, jugando o charlando"), habilidades atléticas ("Alguien bueno en deportes, con buenas habilidades físicas"), compromiso académico ("Alguien que trabaja duro en clase"), empatía ("Alguien que se preocupa por los demás, que es capaz de ponerse en el lugar de otros"), soledad ("Alguien que prefiere estar solo"), autoestima ("Alguien con confianza y seguridad en sí mismo, con buena autoestima"), ansiedad social ("Alguien que parece nervioso o ansioso cuando se relaciona con los demás”), cooperación ("Alguien que coopera bien con los demás, con quien se trabaja bien en grupo"), simpatía (“Alguien simpático, amable"), engreimiento ("Alguien creído, engreído, que se cree superior a los demás"), y divertido (“Alguien con quien se pasa bien, divertido"); (e) Atractivo personal. Se incluyeron dos dimensiones de atracción, el atractivo físico ("Alguien atractivo físicamente") y la Imagen ("Alguien cuya imagen resulta atractiva") y (f) control-permisividad familiar: recogiendo el interés del profesorado de centros de secundaria con los que se contactó, se añadió una cuestión relacionada con la familia, en positivo y negativo: "Alguien a quien ponen muchos problemas en casa para hacer lo que quiere (ir a conciertos, llegar tarde, usar Tuenti o Facebook cuando y como quiera...", que denominamos control familiar $\mathrm{y}$ "Alguien a quien nunca ponen problemas en casa para hacer lo que quiere (ir a conciertos, llegar tarde, usar Tuenti o Facebook cuando y como quiera...)", que denominamos permisividad familiar. Con la introducción de esta dimensión no se pretende disponer de una medida de estilo educativo familiar, sino explorar la asociación entre ausencia de control familiar en relación con determinados aspectos que parecen otorgar estatus entre los iguales.

\section{Nominaciones de Agresión}

Siguiendo a Prinstein y Cillessen (2003) se incluyeron tres tipos de agresión: directa ("Alguien que trata mal a los demás, los amenaza o les hace daño físicamente, por ejemplo, insulta, pone motes, se burla, empuja...”), relacional ("Alguien que usa las relaciones para dañar a otros, por ejemplo, excluyendo a la gente de su grupo de amigos, ignorando o no hablándole a alguien, no invitándole a participar en actividades...") y reputacional (“Alguien que hace cosas para dañar la imagen de otro, por ejemplo, cotillea o expande rumores sobre esa persona, o dice cosas malas de ella a sus espaldas").

Por cada ítem, 27 de estatus y características personales y 3 de agresión se calculó la proporción de nominaciones recibidas por cada ítem. A la variable aceptación social se restó la variable rechazo social, obteniendo la variable pre- 
ferencia social; a la variable popularidad se restó impopularidad, obteniendo la variable popularidad percibida.

\section{RESULTADOS}

Para responder a nuestros objetivos e hipótesis de investigación se analizarán, en primer lugar, la correlación entre preferencia y popularidad percibida, las diferencias de género en ambas dimensiones, así como las correlaciones entre las variables de características personales, atractivo y agresión y, respectivamente, la preferencia social y la popularidad percibida.

En la Tabla 1 se observa que preferencia y popularidad percibida correlacionan claramente entre sí. No existen diferencias significativas en las medias de chicos y chicas, ni para preferencia social $\left(\mathrm{t}_{272}=-1,07, \mathrm{p}=.29\right)$, ni para popularidad $\left(\mathrm{t}_{272}=0,39, \mathrm{p}=.69\right)$.

Ambas dimensiones correlacionan positivamente con características relacionales positivas: ayuda, cooperación, empatía, simpatía, ser divertido y autoestima; también lo hacen con tres de las prerrogativas o ventajas de estatus, es decir, con influencia, admiración y liderazgo, así como con habilidades atléticas, atractivo físico y una imagen atractiva. Ambas dimensiones correlacionan negativamente con tristeza, soledad, ansiedad social.

Sin embargo, se observan también importantes diferencias en el patrón de correlaciones de preferencia y popularidad percibida. Así, la preferencia, y no la popularidad percibida, correlaciona positivamente con la inteligencia y el compromiso académico. La popularidad percibida, por su parte, correlaciona positivamente con la disrupción, la transgresión de normas, el control, el engreimiento y los tres tipos de agresión evaluados, la directa, la relacional y la reputacional; 
también lo hace con permisividad familiar.

\begin{tabular}{|c|c|c|}
\hline & Preferencia & Popularidad Percibida \\
\hline Preferencia & & $.61 * * *$ \\
\hline Ayuda & $.52 * * *$ & $.31 * * *$ \\
\hline Cooperación & $.57 * * *$ & $.29 * * *$ \\
\hline Empatía & $.46^{* * *}$ & $.28 * * *$ \\
\hline Simpatía, Amabilidad & $.62 * * *$ & $.35 * * *$ \\
\hline Diversión & $.57 * * *$ & $.46^{* * *}$ \\
\hline Autoestima & $.51 * * *$ & $.43 * * *$ \\
\hline Inteligencia & $.29 * * *$ & .11 \\
\hline Compromiso Académico & $.27 * * *$ & .08 \\
\hline Influencia & $.41 * * *$ & $.45 * * *$ \\
\hline Control & .02 & $.23 * * *$ \\
\hline Admiración & $.37 * * *$ & $.34 * * *$ \\
\hline Liderazgo & $.30 * * *$ & $.39 * * *$ \\
\hline Habilidades Atléticas & $.20 * * *$ & $.27 * * *$ \\
\hline Atractivo Físico & $.28 * * *$ & $33 * * *$ \\
\hline Imagen Atractiva & $.28 * * *$ & $.32 * * *$ \\
\hline Engreimiento & .08 & $.24 * * *$ \\
\hline Tristeza & $-.39 * * *$ & $-.61 * * *$ \\
\hline Soledad & $-.37 * * *$ & $-.61 * * *$ \\
\hline Ansiedad Social & $-.24 * * *$ & $-.29 * * *$ \\
\hline Transgresión Normas & -.02 & $.24 * * *$ \\
\hline Disrupción & .05 & $.25 * * *$ \\
\hline Agresión Directa & .01 & $.22 * *$ \\
\hline Agresión Relacional & -.03 & $.18^{* *}$ \\
\hline Agresión Reputacional & .01 & $.16^{* *}$ \\
\hline Control Familiar & .04 & -.05 \\
\hline Permisividad familiar & $.14^{*}$ & $.30 * *$ \\
\hline
\end{tabular}

$* * * \mathrm{p}<.001,{ }^{*} \mathrm{p}<.01,{ }^{*} \mathrm{p}<.05$

Tabla 1. Correlaciones entre Preferencia Social, Popularidad Percibida, Características Personales y Agresión.

En segundo lugar, y para responder a nuestro siguiente objetivo, replicamos estas correlaciones para cada género, obteniendo los resultados expuestos en la Tabla 2 (se señalan en negrita las discrepancias). Como se hipotetizaba, aparecen 
distintos patrones correlacionales asociados al género. Así, en relación con la preferencia, el compromiso académico y la permisividad familiar sólo se relaciona con la misma en el caso de las chicas, y las habilidades atléticas sólo en los chicos.

En cuanto a la popularidad percibida, aún se encuentran más diferencias ligadas al género. De nuevo, las habilidades atléticas sólo se relacionan de manera estadísticamente significativa en los chicos, mientras que la inteligencia, el compromiso académico y el engreimiento, en las chicas. Si se observan las variables de agresión, se comprueba que, mientras la agresión directa se relaciona con la popularidad percibida en ambos grupos, la relacional y la reputacional sólo es significativa para las chicas.

\begin{tabular}{|c|c|c|c|c|}
\hline & Chicos & Chicas & Chicos & Chicas \\
\hline & Preferencia & Preferencia & $\begin{array}{c}\text { Popularidad } \\
\text { Percibida }\end{array}$ & $\begin{array}{c}\text { Popularidad } \\
\text { Percibida } \\
\end{array}$ \\
\hline Ayuda & $.42 * * *$ & $.59 * * *$ & $.22 * * *$ & $.38 * * *$ \\
\hline Cooperación & $.45 * * *$ & $.67 * * *$ & $.24 * *$ & $.28 * * *$ \\
\hline Empatía & $.37 * * *$ & $.55 * * *$ & $.24 * *$ & $.35 * * *$ \\
\hline Simpatía & $.59 * * *$ & $.64 * * *$ & $.35 * * *$ & $.36 * * *$ \\
\hline Diversión & $.65 * * *$ & $.49 * * *$ & $.53 * * *$ & $.40 * * *$ \\
\hline Autoestima & $.52 * * *$ & $.50 * * *$ & $.39 * * *$ & $.47 * * *$ \\
\hline Inteligencia & $.19 * * *$ & $.36 * * *$ & -.02 & $.21 * *$ \\
\hline Compromiso Académico & .15 & $.36 * * *$ & -.02 & $.16 * * *$ \\
\hline Influencia & $.43 * * *$ & $.39 * * *$ & $.47 * * *$ & $.45 * * *$ \\
\hline Control & .11 & -.05 & $.29 * * *$ & $.20 *$ \\
\hline Admiración & $.37 * * *$ & $.38 * * *$ & $.31 * * *$ & $.37 * * *$ \\
\hline Liderazgo & $.29 * * *$ & $.31 * * *$ & $.41 * * *$ & $.39 * * *$ \\
\hline Habilidades Atléticas & $.38 * * *$ & .03 & $.39 * *$ & .20 \\
\hline Atractivo Físico & $.35 * * *$ & $.29 * * *$ & $.23 * *$ & $.40 * * *$ \\
\hline Imagen Atractiva & $.37 * * *$ & $.26 * * *$ & $.32 * *$ & $.35 * * *$ \\
\hline Engreimiento & .08 & .09 & .17 & $.33 * *$ \\
\hline Tristeza & $-.32 * * *$ & $-.49 * * *$ & $-.59 * * *$ & $-.67 * * *$ \\
\hline Soledad & $-.28 * * *$ & $-.52 * * *$ & $-.59 * * *$ & $-.71 * * *$ \\
\hline Ansiedad Social & $-.19 * * *$ & $-.27 * * *$ & $-.43 * * *$ & $-.21 * *$ \\
\hline Transgresión Normas & .03 & -.06 & $.25 * *$ & $.25 * *$ \\
\hline Disrupción & .08 & .03 & $.22 *$ & .27 \\
\hline Agresión Directa & .03 & .04 & $.24 * *$ & $.26 * * *$ \\
\hline Agresión Relacional & -.15 & .08 & .09 & $.24 * *$ \\
\hline Agresión Reputacional & .02 & -.02 & .04 & $.24 * *$ \\
\hline Control Familiar & -.01 & .09 & -.07 & -.05 \\
\hline Permisividad familiar & .08 & $.19 * * *$ & $.26 * *$ & $.33 * * *$ \\
\hline
\end{tabular}

$* * * \mathrm{p}<.001, \quad * * \mathrm{p}<.01, \quad * \mathrm{p}<.05$

Psy, Soc, \& Educ, 2011, Vol.3, No1 


\section{Tabla 2. Correlaciones por Género entre Preferencia Social, Popularidad Percibida, Características Personales y Agresión.}

Como se muestra en la Tabla 2, muchas de las variables que correlacionan de forma estadísticamente significativa con las dimensiones de estatus para ambos sexos presentan valores muy diferentes para unas y otros. Para analizar estas diferencias, se realizaron pruebas de contraste $\mathrm{Z}$ entre las correlacionesde chicas y de chicos. Sólo aparecen tres diferencias estadísticamente significativas. En relación con la preferencia, la correlación positiva con la variable diversión es significativamente mayor en chicos $(z=1,94, p=0,026)$, mientras que la asociación negativa con tristeza $(z=1,66, p=0,048)$ y con soledad $(z=2,34, p=0,009)$ es significativamente mayor para las chicas. En lo que respecta a la popularidad, se repite el resultado para soledad $(z=1,69, p=0,045)$.

\section{DISCUSIÓN}

Como se había previsto, y en relación con el primer objetivo del trabajo, preferencia y popularidad percibida resultan dos fenómenos con muchos elementos en común, pero también diferencias relevantes. La correlación que presenta, de .61 muestra claramente su relación, pero índica que no son dimensiones idénticas.

Con respecto a nuestro siguiente objetivo, y coincidiendo también con las hipótesis de partida, la visibilidad social asociada a ambas dimensiones está claramente relacionada con las percepciones por parte del grupo de iguales de características relacionales positivas y atractivas, relacionadas con la conducta prosocial y las habilidades sociales, como la ayuda, la empatía, la cooperación, ser divertido y simpático o disponer de buenos niveles de confianza en uno mismo. Ambas se relacionan también con la percepción grupal de posiciones ventajosas y de poder en el grupo, lo que hemos llamado prerrogativas de estatus, en este caso, influencia, admiración y liderazgo. Otras dimensiones que inciden en el atractivo de quienes son preferidos y populares son las habilidades atléticas, el atractivo físico y una imagen personal atractiva. Por otra parte, ambas dimensiones se alejan de las dificultades en las relaciones ligadas a la tristeza, la soledad, y la ansiedad social.

Las diferencias entre ambas, sin embargo, son también claras. Sólo la preferencia aparece asociada a la percepción de inteligencia y el compromiso académico, mientras comienzan a aparecer características no tan positivas ligadas a la popularidad percibida, esto es, la disrupción, la transgresión de normas o el engreimiento. Si las prerrogativas de estatus se relacionaban, como se señalaba anteriormente, con ambas dimensiones, existe una excepción significativa: el control, probablemente la variable más cercana a la dominancia social tal como es descrita por Hawley (1999), se asocia exclusivamente a la popularidad percibida. Lo mismo ocurre con los tres tipos de agresión evaluados, la directa, la relacional y la reputacional, así como la permisividad familiar. 
Aunque no podamos hablar propiamente de perfiles, pues los análisis son sólo correlacionales, no hay duda de que se vislumbran dos tipologías de prominencia en el grupo bien distintas, y coincidentes con trabajos anteriores (de Bruyn y Cillessen; 2006 a y b; Hawley, 1999; Newcomb, Bukowski y Pattee, 1993; Lease, Kennedy y Axelroad, 2002; Lease, Musgrove y Axelroad, 2002).El primero de los tipos, vinculado a la preferencia, parece acumular mayor cantidad de aspectos valorados en el proceso de socialización por parte de los adultos. El segundo, vinculado a la popularidad, mantiene algunos componentes positivos en común con el primero, añadiendo otros componentes cuestionables desde el punto de vista de la socialización, aunque especialmente atractivos a estas edades por ser percibidos como indicadores de autonomía frente a los adultos, como podría ocurrir con la transgresión a las normas.

Las comparaciones por género, nuestro segundo objetivo, permiten perfilar aún mejor las diferencias. Parece claro que algunas de las discrepancias que hemos analizado entre preferencia y popularidad percibida son, en realidad, diferencias de género. Así, el compromiso académico solo se relacionaría con la preferencia en las chicas, y la permisividad familiar solo sería importante para ellas. Las habilidades atléticas, sin embargo, es una variable significativa solo para la preferencias de los chicos. En cuanto a la popularidad, las habilidades atléticas siguen siendo una variable de los chicos, y lo mismo ocurre con una de las variables negativas a las que hacíamos referencia, la disrupción. Mientras, la inteligencia solo resulta significativa para las chicas, pero también el engreimiento. El compromiso académico, que no aparecía relacionado con la popularidad en los datos globales, aparece, sin embargo, correlacionado significativamente con la popularidad en las chicas. Del mismo modo resulta interesante como la relación encontrada entre los tipos de agresión y la popularidad se perfila al introducir el género: solo en las chicas tiene relevancia la agresividad relacional y la reputacional. Estas diferencias de género parecen enfatizar para el caso de las chicas la conexión entre preferencia y características valoradas en el proceso de socialización, indicando una mayor presión socializadora hacia características relacionadas con el compromiso académico en las chicas, extendiéndose esta valoración de lo académico para ellas a la popularidad. Por su parte, el perfil de popularidad de los chicos acumularía mayor número de aspectos negativos.

Pese a lo interesante de las discrepancias, con estos datos, y con las debidas precauciones dada la naturaleza de este trabajo, cabe destacar un aspecto relevante en torno a las diferencias de género en estos patrones correlacionales. Así, aunque las diferencias de género en algunas de estas dimensiones están muy establecidas, como sería el caso de la empatía, la ayuda, la cooperación o la agresión directa, tanto específicamente en reputación (Muñoz Tinoco, Jiménez-Lagares y Moreno, 2008; Vysniauskyte-Rimkiene y Kardelis, 2005), como a través de trabajos con un enfoque más amplio de diferencias de género en conducta social (Aunola, Stattin y Nurmi, 2000; Galambos,2004; Lindeman, Harakka y Keltikangas-Järvinen,1997; Lemos, Fidalgo, Calvo y Menéndez, 1992; Maccoby,2004, Pakaslahti, Karjalainen, y Keltikangas-Järvinen, 2006), no sólo 
mantienen correlaciones significativas en ambos géneros, sino que el tamaño de la correlación es estadísticamente similar para ambos sexos, como indican los contrastes z utilizados. Las excepciones, con respecto a estos últimos contrastes, de las variables tristeza y soledad con correlaciones negativas significativamente mayores en chicas merecerían, sin duda, análisis posteriores que incidan en las relaciones entre el género y la dimensión de aislamiento.

En cualquier caso, parece claro que para entender de modo completo las diferencias entre preferencia y popularidad percibida resulta ineludible contemplar el género, ya que variables aparentemente relevantes aparecen asociadas exclusivamente a uno de los géneros. Así, resultaría impreciso hablar de que la competencia académica se relaciona con la preferencia, pues sólo ocurre en chicas, o señalar que las habilidades atléticas se relacionan tanto con la preferencia como con la popularidad, porque sólo ocurre para los chicos en ambos casos.

Como se señaló en páginas anteriores, este trabajo intenta ser una primera aproximación a un tema prácticamente inédito en nuestro entorno, de ahí su carácter descriptivo y exclusivamente correlacional. Trabajos posteriores deberían ahondar en la contribución específica de las distintas variables estudiadas a la preferencia y la popularidad, y el papel mediador o modulador del sexo; la clasificación de chicos y chicas con respecto a ambas dimensiones, y los perfiles conductuales específicos de los distintos grupos de clasificación (Lease, Kennedy y Axelroad, 2002); o el análisis de la posible heterogeneidad dentro de los grupos de clasificación (de Bruyn y Cillessen, 2006; Lease, Kennedy y Axelroad, 2002).

\section{REFERENCIAS}

Adler, P. A., y Adler, P. (1998). Peer power: Preadolescent culture and identity. New Jersey: Rutgers University Press.

Aunola, K., Stattin, H. y Nurmi, J. (2000). Adolescents' achievement strategies,school adjustment, and externalizing and internalizing problembehaviors.Journal of Youth and Adolescence, 29(3), 289-305.

Cillessen, A. H. N., y Rose, A. J. (2005). Understanding popularity in the peer system.Current Directions in Psychological Science, 14(2), 102.

Coie, J. D., Dodge, K. A., yKupersmidt, J. B. (1990). Peer group behavior and social status. En R. Asher y J. D. Coie (Eds.), Peer rejection in childhood (pp.17-59). New York: Cambridge University Press.

De Bruyn, E. H., y Cillessen, A. H. N. (2006a). Popularity in early adolescence: Prosocial and antisocial subtypes. Journal of Adolescent Research, 21(6), 607.

De Bruyn, E. H., y Cillessen, A. H. N. (2006b). Heterogeneity of girls' consensual popularity: Academic and interpersonal behavioral profiles. Journal of Youth and Adolescence, 35(3), 412-422.

Emler, N. (1990). A social psychology of reputation. European review of social psychology, 1(1), 171-193.

Estell, D. B., Farmer, T. W., Pearl, R., Van Acker, R., yRodkin, P. C. (2003). 
Heterogeneity in the relationship between popularity and aggression: Individual, group, and classroom influences. New Directions for Child and Adolescent Development, 101, 75-85.

Galambos, N. L. (2004). Gender and gender role development in adolescence.En R. M. Lerner y L. Steinberg (Eds.), Handbook of adolescent psychology (pp. 233-262).New Jersey: Wiley.

García-Bacete, F. J. (2008). Identificación de subtipos sociométricos en niños y niñas de 6 a 11 años. Revista Mexicana de Psicología, 25(2), 209-222.

Gest, S. D., Sesma, A., Masten, A. S., y Tellegen, A. (2006). Childhood peer reputation as a predictor of competence and symptoms 10 years later. Journal of abnormal child psychology, 34(4), 507-524.

Hawley, P. H. (1999). The ontogenesis of social dominance: A strategy-based evolutionary perspective. Developmental Review, 19, 97-132.

Lease, A. M., Kennedy, C. A., y Axelrod, J. L. (2002).Children's social constructions of popularity.Social Development, 11(1), 87-109.

Lease, A. M., Musgrove, K. T., y Axelrod, J. L. (2002). Dimensions of social status in preadolescent peer groups: Likability, perceived popularity, and social dominance. Social Development, 11(4), 508-533.

Lemos, S., Fidalgo, A. M., Calvo, P. y Menéndez, P. (1992). Salud mental delos adolescentes asturianos. Psicothema, 4(1), 21-48.

Maccoby, E. E. (2004). Aggresion in the context of gender development.En M. Putallaz y K. L. Bierman (Eds.), Aggresion, antisocial behavior, and violence among girls (pp. 3-22). London: The Guilford Press.

Miller-Johnson, S., Costanzo, P. R., Coie, J. D., Rose, M. R., Browne, D. C., y Johnson, C. (2003). Peer social structure and risk-taking behaviors among African American early adolescents. Journal of Youth and Adolescence, 32(5), 375-384.

Newcomb, A. F., Bukowski, W. M., y Pattee, L. (1993). Children's peer relations: A meta-analytic review of popular, rejected, neglected, controversial, and average sociometric status. Psychological Bulletin, 113(1), 99.

Parker, J. G., y Asher, S. R. (1987). Peer relations and later personal adjustment: Are low-accepted children at risk? Psychological Bulletin, 102(3), 357-389.

Pakaslahti, L., Karjalainen, A. y Keltikangas-Järvinen, L. (2006). Relationshipsbetween adolescent prosocial problem-solving strategies, prosocialbehaviour, and social acceptance.International Journal of Behavioral Development, 26(2), 137-144.

Parkhurst, J. T., y Hopmeyer, A. (1998). Sociometric popularity and peer-perceived popularity.The Journal of Early Adolescence, 18(2), 125.

Prinstein, M. J., y Cillessen, A. H. (2003). Forms and functions of adolescent peer aggression associated with high levels of peer status. Merrill-Palmer Quarterly, 49(3), 310-342.

Rodkin, P. C., Farmer, T. W., Pearl, R., y Van Acker, R. (2000). Heterogeneity of popular boys: Antisocial and prosocial configurations. Developmental Psychology, 36(1), 14. 
Rubin, K. H., Bukowski, W., y Parker, J. G. (1998). Peer interactions, relationships, and groups. En Handbook of Child Psychology, Vol. 3, (pp. 619-700). New Jersey: Wiley

Rubin, K. H., Bukowski, W., y Parker, J. G. (2006). Peer interactions, relationships, and groups. Handbook of Child Psychology, Vol 3, (pp. 619-700). New Jersey: Wiley

Vysniauskyte-Rimkiene, J., y Kardelis, K. (2005). Peer reputation of adolescents: sociometric status differences. Medicina (Kaunas), 41(6), 522-528.

Andreou, E. (2006). Social preference, perceived popularity and social intelligence. School Psychology International, 27(3), 339.

Hymel, S., Wagner, E., \& Butler, L. J. (1990). Reputational bias: View from the peer group. En R. Asher y J. D. Coie (Eds.), Peer rejection in childhood (pp.17-59). New York: Cambridge University Press

Lindeman, M., Harakka, T., \& Keltikangas-Järvinen, L. (1997). Age and gender differences in adolescents' reactions to conflict situations: Aggression, prosociality, and withdrawal. Journal of Youth and Adolescence, 26(3), 339-351.

Muñoz Tinoco, M. V., Jiménez Lagares, I., y Moreno Rodríguez, M. C. (2008). Reputación conductual y género en la adolescencia. Anales de psicología, 24(2), 334-340. 